\title{
Regge trajectories for the mesons consisting of different quarks
}

\author{
Jiao-Kai Chen ${ }^{\mathrm{a}}$ \\ School of Physics and Information Science, Shanxi Normal University, Linfen 041004, China
}

Received: 8 July 2018 / Accepted: 3 August 2018 / Published online: 14 August 2018

(C) The Author(s) 2018

\begin{abstract}
By applying the Bohr-Sommerfeld quantization approach to the quadratic form of the spinless Salpeter-type equation (QSSE), we show that the obtained Regge trajectories for the mesons consisting of unequally massive quarks take the form $M^{2}=\beta\left(c_{l} l+\pi n_{r}+c_{0}\right)^{2 / 3}+c_{1}$, which have the same form as the Regge trajectories for charmonia and bottomonia. Then we apply the obtained Regge trajectories to fit the spectra of the strange mesons, the heavy-light mesons (the $D, D_{s}, B$ and $B_{s}$ mesons) and the bottom-charmed mesons. The fitted Regge trajectories are in agreement with the experimental data and the theoretical predictions, which demonstrates that the newly proposed Regge trajectories can be applied universally to the light mesons, the heavy-light mesons and the heavy mesons. By fitting the spectra of the mesons composed of different quarks, the concavity of these Regge trajectories are illustrated, which is of cardinal significance for the potential models.
\end{abstract}

\section{Introduction}

The Regge trajectory is one of the effective approaches for studying hadron spectra [1-22]. For one newly proposed Regge trajectories, checking its universality is an important and necessary task. In Ref. [23], the authors applied the rotational states of the string with massive ends including the spin-orbit correction to describe the Regge trajectories for the light, strange, charmed, bottom mesons, and for the $\Delta / \Sigma / \Lambda / \Lambda_{c}$ baryons. In Ref. [24], the authors used the Regge-like formula $\left(M-m_{Q}\right)^{2}=\pi \sigma L$ to analyze all the heavy-light mesons and the baryons which can be assumed consisting of one heavy quark and one light cluster of two light quarks.

In Ref. [25], we proposed one new form of the Regge trajectories,
$M^{2}=\beta\left(c_{l} l+\pi n_{r}+c_{0}\right)^{2 / 3}+c_{1}$,

where $M$ is the meson mass, $l$ is the orbital angular momentum, $n_{r}$ is the radial quantum number. $\beta$ and $c_{l}$ are universal parameters. $c_{0}$ and $c_{1}$ vary with different trajectories. As applying the formula (1) to fit the bottomonia and charmonia, the results are excellent. In the present work, we use the quadratic form of the spinless Salpeter-type equation (QSSE) [26-33] to discuss the Regge trajectories for the mesons consisting of different quarks. We find that the obtained formula can be written in the same form as the Regge trajectories in Eq. (1). The fitted Regge trajectories agree with the experimental data and the theoretical values. By fitting data, we notice that the Regge trajectories for these meson are concave, which is of cardinal significance for the potential models because this property of the Regge trajectories can assist in the choice of the appropriate dynamic equation and potential to describe mesons.

This paper is organized as follows. In Sect. 2, the new form of the Regge trajectories for mesons composed of different quarks is obtained from the QSSE, which has the same form as that for heavy quarkonia. In Sect. 3, the obtained Regge trajectory is applied to fit the spectra of the strange mesons, the heavy-light mesons and the bottom-charmed mesons. In Sect. 4, the universality and concavity of the newly proposed Regge trajectories are discussed. We conclude in Sect. 5.

\section{Regge trajectories from the QSSE}

In this section, the QSSE is briefly reviewed at first. Then the orbital and radial Regge trajectories for the mesons consisting of unequally massive quarks are obtained from the QSSE by employing the Bohr-Sommerfeld quantization approach $[13,34]$, which have the same form as the Regge trajectories for heavy quarkonia obtained in Ref. [25].

a e-mails: chenjk@sxnu.edu.cn;chenjkphy@outlook.com 


\subsection{QSSE}

It is well known that the Bethe-Salpeter equation $[35,36]$ is an appropriate tool to deal with bound states. In Ref. [27], the authors obtained a first principal Bethe-Salpeter equation, and then reduced it to the eigenvalue equation for the square mass operator [26-30] by means of a three dimensional reduction

$M^{2}=M_{0}^{2}+U$,

where

$M_{0}=\omega_{1}+\omega_{2}=\sqrt{m_{1}^{2}+\mathbf{p}^{2}}+\sqrt{m_{2}^{2}+\mathbf{p}^{2}}$,

$\left\langle\mathbf{p}|U| \mathbf{p}^{\prime}\right\rangle=\frac{1}{(2 \pi)^{3}} \sqrt{\frac{w_{1}+w_{2}}{2 w_{1} w_{2}}} \hat{I}_{a b}^{\text {inst }}\left(\mathbf{p}, \mathbf{p}^{\prime}\right) \sqrt{\frac{w_{1}^{\prime}+w_{2}^{\prime}}{2 w_{1}^{\prime} w_{2}^{\prime}}} \sigma_{1}^{a} \sigma_{2}^{b}$.

In the above equations, $M$ is the bound state mass, $\mathbf{p}$ the c.m. momentum of quarks, $m_{1}$ and $m_{2}$ their constituent masses, $\omega_{1}^{\prime}=\sqrt{m_{1}^{2}+\mathbf{p}^{\prime 2}}$ and $\omega_{2}^{\prime}=\sqrt{m_{2}^{2}+\mathbf{p}^{\prime 2}}$. $\hat{I}_{a b}^{\text {inst }}\left(\mathbf{p}, \mathbf{p}^{\prime}\right)$ is the instantaneous kernel. Neglecting any reference to the spin degrees of freedom of the involved bound-state constituents, Eq. (2) reduces to the QSSE which is written in configuration space as [29-33]

$M^{2} \Psi(\mathbf{r})=\left[\omega_{1}+\omega_{2}\right]^{2} \Psi(\mathbf{r})+\mathcal{U} \Psi(\mathbf{r})$,

where $\omega_{i}$ is the square-root operator of the relativistic kinetic energy of constituent

$\omega_{i}=\sqrt{m_{i}^{2}-\Delta}$.

$\Delta$ is the Laplacian. Let $m_{1} \geq m_{2}$, there is the inequality for $M_{0}^{2}$

$4\left(m_{2}^{2}+\mathbf{p}^{2}\right) \leq M_{0}^{2} \leq 4\left(m_{1}^{2}+\mathbf{p}^{2}\right)$.

In case of $|\mathbf{p}| \gg m_{1}, m_{2}$, there is

$M_{0}^{2} \approx 4 \mathbf{p}^{2}+2\left(m_{1}^{2}+m_{2}^{2}\right)$.

In case of $|\mathbf{p}| \ll m_{1}, m_{2}$, there is

$M_{0}^{2} \approx\left(2+\frac{m_{1}}{m_{2}}+\frac{m_{2}}{m_{1}}\right) \mathbf{p}^{2}+\left(m_{1}+m_{2}\right)^{2}$.

For simplicity, we assume that $\mathcal{U}$ takes the following form

$\mathcal{U}=-\frac{A}{r}+B r$

which is a variant of the well-known Cornell potential [37]. $A$ and $B$ vary with the discussed mesons. For example, $A=$ $2\left(m_{b}+m_{c}\right) \alpha$ and $B=2\left(m_{b}+m_{c}\right) \sigma$ for the bottom-charmed mesons, where $m_{c}$ and $m_{b}$ are the charm quark mass and the bottom quark mass, respectively. $\alpha=\alpha_{s} 4 / 3, \alpha_{s}$ is the strong coupling constant of the color Coulomb interaction. $\sigma$ is the string tension.
2.2 Regge trajectories for the mesons composed of different quarks

Due to the $\omega_{1} \omega_{2}$ term, the Regge trajectories can not be obtained directly from Eq. (4). Using Eqs. (4), (6) and (7), we propose two auxiliary equations

$$
\begin{gathered}
M_{i}^{2} \Psi(\mathbf{r})=4\left(m_{i}^{2}+\mathbf{p}^{2}\right) \Psi(\mathbf{r})+\left(-\frac{A}{r}+B r\right) \Psi(\mathbf{r}), \\
i=1,2,
\end{gathered}
$$

where

$\mathbf{p}^{2}=p_{r}^{2}+\frac{p_{\phi}^{2}}{r^{2}}$.

Following Refs. [13,25] to employ the Bohr-Sommerfeld quantization approach [34], the orbital Regge trajectories and the radial Regge trajectories can be easily obtained from Eqs. (8),

$M_{i}^{2} \sim \beta_{l} l^{2 / 3}, \quad \beta_{l}=3 B^{2 / 3}$,

$M_{i}^{2} \sim \beta_{n_{r}} n_{r}^{2 / 3}, \quad \beta_{n_{r}}=(3 \pi B)^{2 / 3}$.

In case of the power-law potential $V(r)=B r^{a}(a>0)$, the orbital Regge trajectories for large $l$ are

$M_{i}^{2} \sim \beta_{l}(a) l^{2 a /(a+2)} \quad(l \gg n)$,

where $\beta_{l}(a)$ reads

$\beta_{l}(a)=2^{2 a /(a+2)} B^{2 /(a+2)}\left(1+\frac{a}{2}\right)\left(\frac{2}{a}\right)^{a /(a+2)}$.

The radial Regge trajectories are

$M^{2} \sim \beta_{n_{r}}(a) n_{r}^{2 a /(a+2)}\left(n_{r} \gg l\right)$.

The Regge slope is

$\beta_{n_{r}}(a)=2^{2 a /(a+2)} B^{2 /(a+2)}\left[\frac{a \pi}{B(1 / a, 3 / 2)}\right]^{2 a /(a+2)}$,

where $B(x, y)$ is the beta function [38].

Using Eqs. (4), (6), (8) and (10), we assume that for Eq. (4) with the potential (7) the radial Regge trajectories are of the form

$M^{2}=\beta_{n_{r}}\left(n_{r}+c_{0}^{\prime}\right)^{2 / 3}+c_{1}^{\prime}$,

and the orbital Regge trajectories take the form

$M^{2}=\beta_{l}\left(l+c_{0}^{\prime \prime}\right)^{2 / 3}+c_{1}^{\prime \prime}$.

By considering Eqs. (15) and (16), we find that the Regge trajectories for mesons composed of different quarks can be rewritten in the same form as the Regge trajectories for heavy quarkonia [25], see Eq. (1).

In Ref. [25], we have shown that the new form of the Regge trajectories [Eq. (1)] are appropriate to the heavy quarkonia. In this work, we show that Eq. (1) can also be appropriate to 


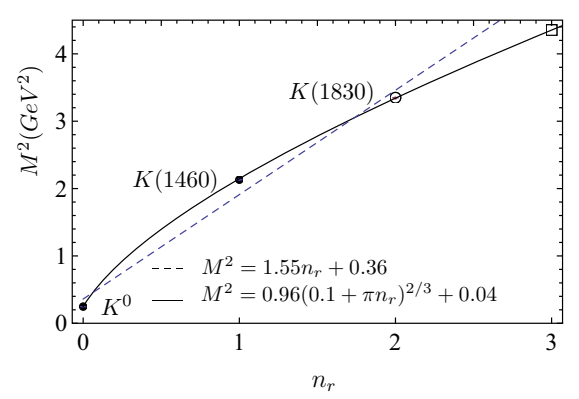

(a)

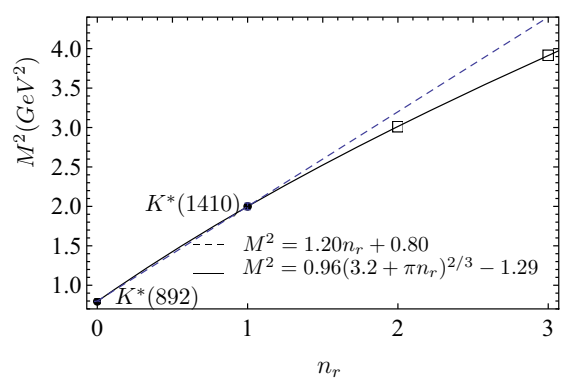

(b)

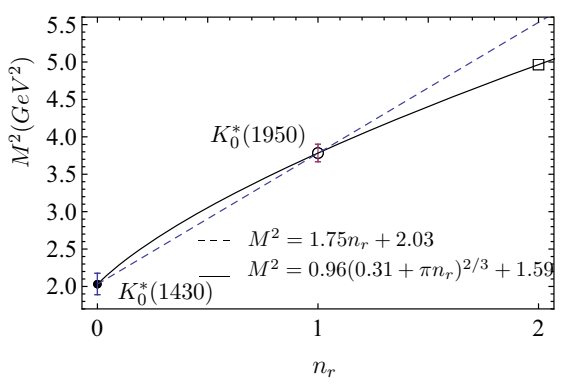

(c)

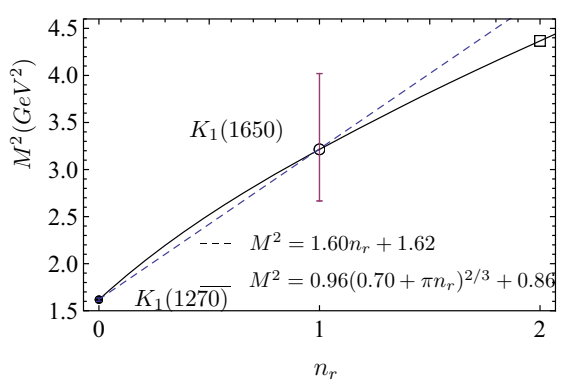

(d)

Fig. 1 The radial Regge trajectories for the strange mesons. The used data are listed in Table 1. The well-established states are given by the solid dots. The unwell-established states or the states needing confir-

the mesons consisting of different quarks. The new form of the Regge trajectories is expected to be universal for mesons. The good fit by employing Eq. (1) illustrates this conclusion.

\section{Regge trajectories for the mesons constituting of different quarks}

In this section, we employ the Regge trajectory formula $M^{2}=\beta\left(c_{l} l+\pi n_{r}+c_{0}\right)^{2 / 3}+c_{1}$ [Eq. (1)] to fit the spectra of the strange mesons, the heavy-light mesons and the bottomcharmed mesons. The parameters of the Regge trajectories are obtained by fitting the experimental data or theoretical values. The fitted radial and orbital Regge trajectories are in agreement with the experimental data or the theoretical predictions. The linear Regge trajectories are also given and we find that the new form of the Regge trajectories are better than the linear ones.

\subsection{Strange mesons}

The universal parameter $\beta$ is calculated by fitting the radial Regge trajectories for $K^{0}$ and $K^{*}(892), \beta=0.96$ for the strange mesons. The universal parameter $c_{l}$ is obtained by fitting the orbital Regge trajectories for $K^{*}(892), c_{l}=4.47$. $c_{0}$ and $c_{1}$ vary with different Regge trajectories. The fitted radial and orbital Regge trajectories are shown in Figs. 1

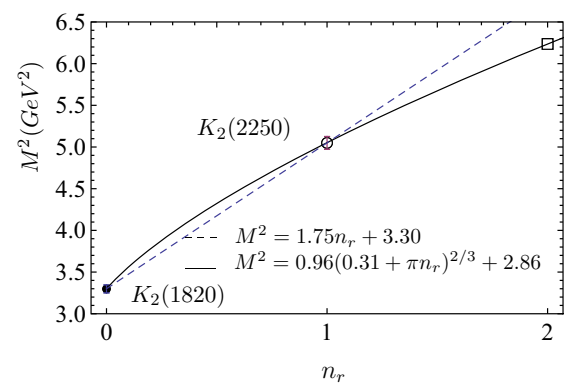

(e)

mation are given by circles. Open squares are predicted masses by the fitted Regge trajectories. The dashed lines are the fitted linear Regge trajectories and the solid lines are the fitted curves by using Eq. (1)

and 2. The fitted and predicted masses of the strange mesons by the Regge trajectories are listed in Table 1 and they are in good agreement with the experimental data and the theoretical values.

$K(1830)$ is suggested to be the $3^{1} S_{0}$ state $[41,43,44]$. $K_{0}^{*}$ (1950) is taken as the $2^{3} P_{0}$ state [41] and $K_{2}(2250)$ as a $2 D_{2}$ state $[17,41]$. The mass of $K_{1}(1650)$ is $1793 \pm 59_{-101}^{+153}$ $\mathrm{MeV}$ [42] and $K_{1}(1650)$ is in favor of a $2 P$ state [41]. In Refs. [41,43], $K_{2}^{*}(1980)$ is assumed to be the $2^{3} P_{2}$ state or the $1^{3} F_{2}$ sate [28]. As $K_{2}^{*}(1980)$ is treated as $1^{3} F_{2}$ sate, the dominant decay channel is shown to be $K_{1}(1270) \pi$ which is not observed in experiments. $K_{4}(2500)$ is the possible candidate of the $2 G$ state $[17,41]$ and the fitted value is in accordance with the experimental data. $K_{5}^{*}(2380)$ is assigned as the $1^{3} G_{5}$ state and needs confirmation [39]. Its mass is larger than the fitted value.

\subsection{Charmed mesons}

By fitting the radial Regge trajectories for $D^{0}$ and $D^{*}(2007)^{0}$, the universal parameter $\beta$ is calculated, $\beta=1.89$ for the charmed mesons. $c_{l}$ is obtained by fitting the orbital Regge trajectories for $D^{0}, D^{*}(2007)^{0}$ and $D_{0}^{*}(2400)^{0}, c_{l}=3.02$. $c_{0}$ and $c_{1}$ vary with different Regge trajectories. In Fig. 3 are the fitted radial and orbital Regge trajectories for the charmed mesons. The fitted and predicted masses of the charmed mesons by the Regge trajectories are listed in Table 2 and 


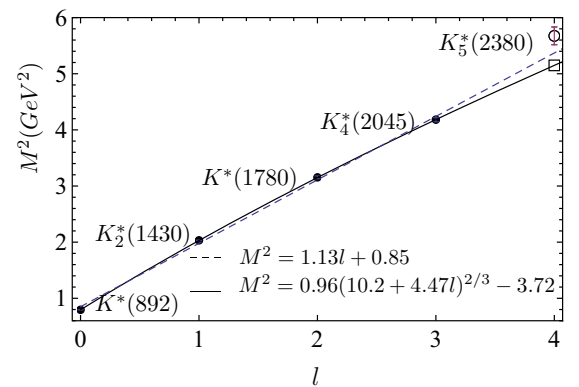

(a)

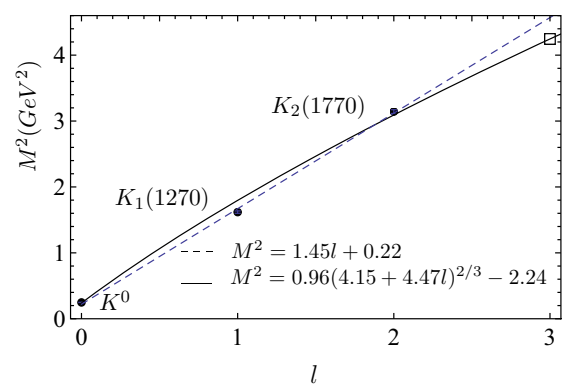

(b)

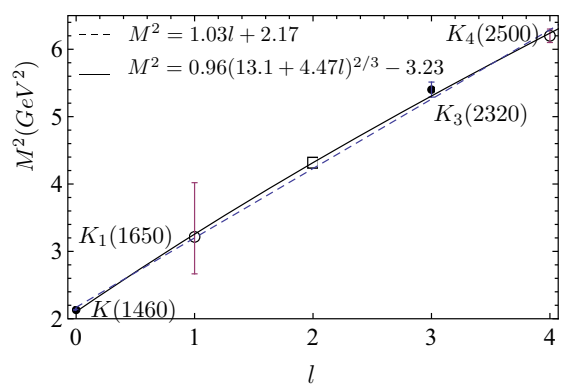

(c)

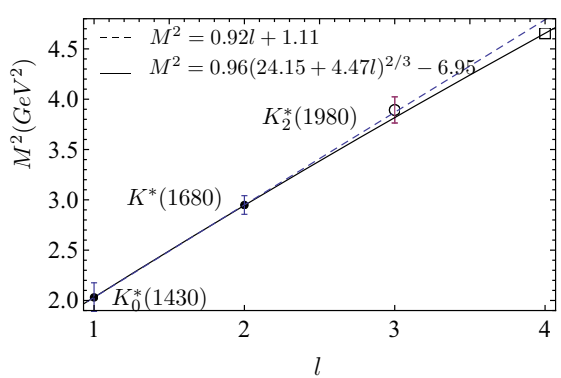

(d)

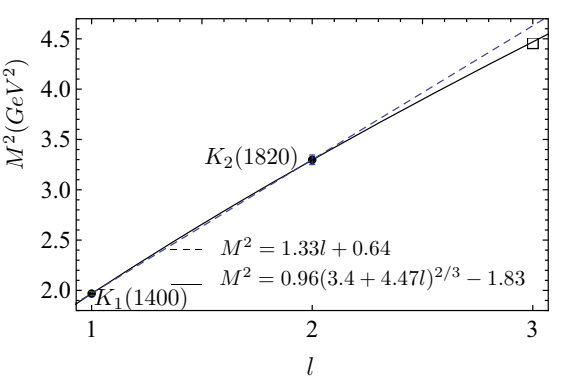

(e)

Fig. 2 Same as Fig. 1 except for the orbital Regge trajectories

Table 1 Masses of the strange mesons (in MeV). The experimental data are from PDG [39]. The fitted results by using the Regge trajectories [Eq. (1)] are shown in comparison with the theoretical values of GI [40], EFG [17] and PWLM [41]. ? denotes the possible candidates for the unwell-established states or the mesons needing confirmation. FO and FR denote the fitted results by the orbital Regge trajectories and by the radial Regge trajectories, respectively

\begin{tabular}{|c|c|c|c|c|c|c|c|c|}
\hline State & $J^{P}$ & Meson & PDG [39] & GI [40] & EFG [17] & PWLM [41] & FR & FO \\
\hline $1^{1} S_{0}$ & $0^{-}$ & $K^{0}$ & $497.611 \pm 0.013$ & 461.5 & 482 & 497.7 & 497 & 489 \\
\hline $2^{1} S_{0}$ & $0^{-}$ & $K(1460)$ & 1460 & 1454 & 1538 & 1457 & 1464 & 1451 \\
\hline $3^{1} S_{0}$ & $0^{-}$ & $K(1830) ?$ & 1830 & 2065 & 2065 & 1924 & 1829 & \\
\hline $4^{1} S_{0}$ & $0^{-}$ & & & & & 2248 & 2087 & \\
\hline $1^{3} S_{1}$ & $1^{-}$ & $K^{*}(892)$ & $891.66 \pm 0.26$ & 902.8 & 897 & 896 & 891 & 892 \\
\hline $2^{3} S_{1}$ & $1^{-}$ & $K^{*}(1410)$ & $1414 \pm 15$ & 1579 & 1675 & 1548 & 1414 & \\
\hline $3^{3} S_{1}$ & $1^{-}$ & & & 2156 & 2156 & 1983 & 1735 & \\
\hline $4^{3} S_{1}$ & $1^{-}$ & & & & & 2287 & 1979 & \\
\hline $1^{3} P_{0}$ & $0^{+}$ & $K_{0}^{*}(1430)$ & $1425 \pm 50$ & 1234 & 1362 & 1257 & 1425 & 1426 \\
\hline $2^{3} P_{0}$ & $0^{+}$ & $K_{0}^{*}(1950) ?$ & $1945 \pm 10 \pm 20$ & 1890 & 1791 & 1829 & 1945 & \\
\hline $3^{3} P_{0}$ & $0^{+}$ & & & 2160 & 2160 & 2176 & 2228 & \\
\hline $1^{1} P_{1}$ & $1^{+}$ & $K_{1}(1270)$ & $1272 \pm 7$ & 1352 & 1294 & 1364 & 1272 & 1340 \\
\hline $2^{1} P_{1}$ & $1^{+}$ & $K_{1}(1650) ?$ & $1793 \pm 59_{-101}^{+153}[42]$ & 1897 & 1757 & 1840 & 1793 & 1805 \\
\hline $3^{1} P_{1}$ & $1^{+}$ & & & 2164 & 2164 & 2177 & 2090 & \\
\hline $1^{3} P_{1}$ & $1^{+}$ & $K_{1}(1400)$ & $1403 \pm 7$ & 1366 & 1412 & 1377 & & 1403 \\
\hline $1^{3} P_{2}$ & $2^{+}$ & $K_{2}^{*}(1430)$ & $1425.6 \pm 1.5$ & 1428 & 1424 & 1431 & & 1426 \\
\hline $1^{3} D_{1}$ & $1^{-}$ & $K^{*}(1680)$ & $1717 \pm 27$ & 1776 & 1699 & 1766 & & 1716 \\
\hline $1^{1} D_{2}$ & $2^{-}$ & $K_{2}(1770)$ & $1773 \pm 8$ & 1791 & 1709 & 1778 & & 1758 \\
\hline $2^{1} D_{2}$ & $2^{-}$ & & & 2238 & 2066 & 2121 & & 2078 \\
\hline $1^{3} D_{2}$ & $2^{-}$ & $K_{2}(1820)$ & $1816 \pm 13$ & 1804 & 1824 & 1789 & 1817 & 1816 \\
\hline $2^{3} D_{2}$ & $2^{-}$ & $K_{2}(2250) ?$ & $2247 \pm 17$ & 2254 & 2163 & 2131 & 2248 & \\
\hline $3^{3} D_{2}$ & $2^{-}$ & & & & & 2388 & 2497 & \\
\hline
\end{tabular}


Table 1 continued

\begin{tabular}{llllllll}
\hline State & $J^{P}$ & Meson & PDG [39] & GI [40] & EFG [17] & PWLM [41] & FR \\
\hline $1^{3} D_{3}$ & $3^{-}$ & $K_{3}^{*}(1780)$ & $1776 \pm 7$ & 1794 & 1789 & 1781 & 1775 \\
$1^{3} F_{2}$ & $2^{+}$ & $K_{2}^{*}(1980) ?$ & $1973 \pm 8 \pm 25$ & 2151 & 1964 & 2093 & 1954 \\
$1^{1} F_{3}$ & $3^{+}$ & & & 2131 & 2009 & 2075 & 2060 \\
$2^{1} F_{3}$ & $3^{+}$ & $K_{3}(2320)$ & $2324 \pm 24$ & 2524 & 2348 & 2340 & 2303 \\
$1^{3} F_{3}$ & $3^{+}$ & & & 2143 & 2080 & 2084 & 2114 \\
$1^{3} F_{4}$ & $4^{+}$ & $K_{4}^{*}(2045)$ & $2045 \pm 9$ & 2108 & 2096 & 2058 & 23045 \\
$1^{1} G_{4}$ & $4^{-}$ & & & 2422 & 2255 & 2309 & 2303 \\
$2^{1} G_{4}$ & $4^{-}$ & $K_{4}(2500) ?$ & $2490 \pm 20$ & 2779 & 2575 & 2520 & 2354 \\
$1^{3} G_{4}$ & $4^{-}$ & & & 2433 & 2285 & 2317 & 2286 \\
$1^{3} G_{5}$ & $5^{-}$ & $K_{5}^{*}(2380) ?$ & $2382 \pm 14 \pm 19$ & 2388 & 2356 & & 2286 \\
\hline
\end{tabular}

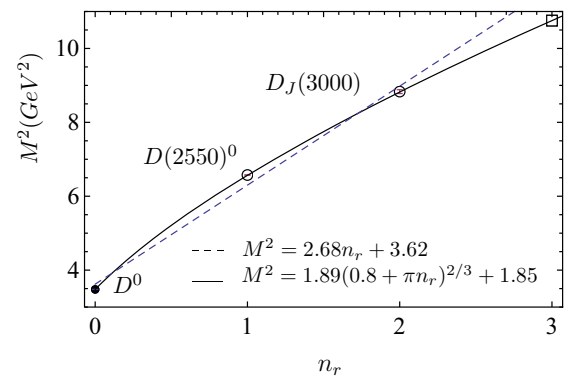

(a)

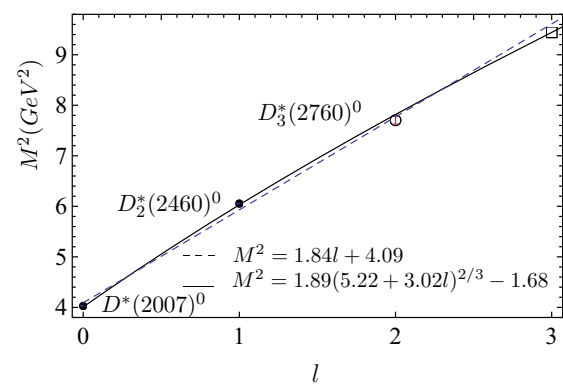

(d)

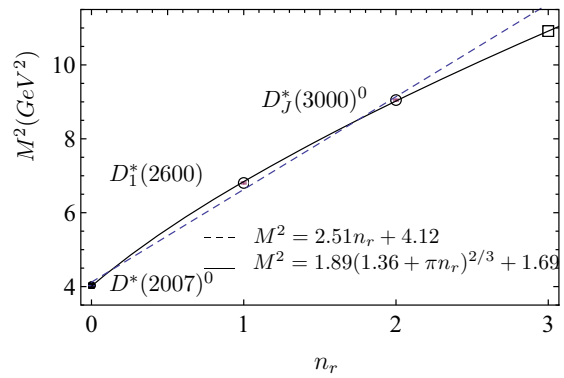

(b)

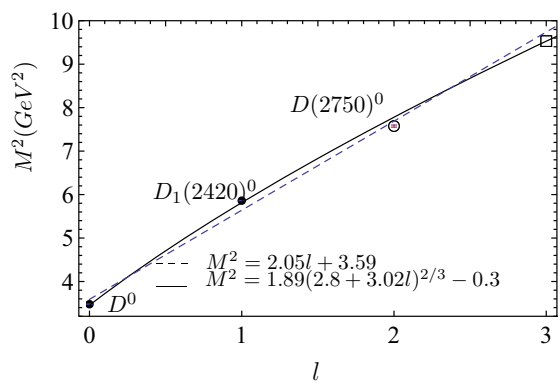

(e)

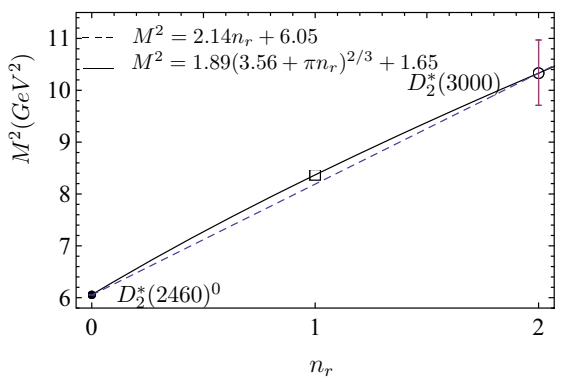

(c)

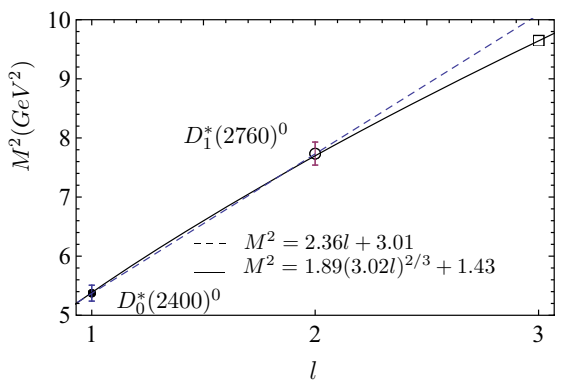

(f)

Fig. 3 The radial and orbital Regge trajectories for the charmed mesons. The used data are listed in Table 2. The well-established states are given by solid dots. The unwell-established states or the states needing confirmation are given by circles. Open squares are predicted masses by the fitted Regge trajectories

they are consistent with the experimental data and the theoretical values.

$D(2550)$ is taken as a candidate for the $2^{1} S_{0}$ state by the helicity distribution analysis [45], and $D_{J}(2580)$ is assumed to be the same state because the resonance parameters of $D_{J}(2580)$ and $D(2550)$ are similar and BaBar assignment [45] is consistent with the LHCb results [46]. $D_{J}(3000)$ is a possible candidate for the $2 P$ state [47], the $3^{1} S_{0}$ state [48], or the $3^{+}$state [49]. $D^{*}(2600)$ can be assigned to be the $2^{3} S_{1}$ state [48,50], see Ref. [51] for more discussions. $D_{J}^{*}(3000)^{0}$ can be the candidate of the $3^{3} S_{1}$ state [51]. $D_{2}^{*}(3000)$ is possibly the $3^{3} P_{2}$ state while the assignment of the $2^{3} F_{2}$ state can not be fully excluded [52]. $D(2750)^{0}$ is assigned as the
$1 D_{2}$ state $[53,54] . D_{1}^{*}(2760)$ and $D_{3}^{*}(2760)$ are assumed to be the $1^{3} D_{1}$ state and $1^{3} D_{3}$ state [53,55-59], respectively.

\subsection{Charmed-strange mesons}

Due to insufficient experimental data, the radial Regge trajectory for the charmed-strange mesons are obtained by fitting the theoretical values in Ref. [60], $\beta=1.75$, see Fig. 4a. The orbital Regge trajectories by fitting the experimental data and by fitting the theoretical values in Ref. [60] are in consistence, see Fig. 4b. The universal parameter is calculated, $c_{l}=2.44$. The experimental data and the theoretical predictions are listed in Table 3. 
Table 2 Masses of the charmed mesons (in MeV). The experimental data are from PDG [39]. The theoretical values are from EFG [16], GM [53], KDR [54] and LNR [60]. ? denotes the possible candidates for the unwell-established states or the mesons needing confirmation. FO and FR denote the fitted results by the orbital Regge trajectories and by the radial Regge trajectories, respectively

\begin{tabular}{|c|c|c|c|c|c|c|c|c|c|}
\hline State & $J^{P}$ & Meson & PDG [39] & EFG [16] & GM [53] & KDR [54] & LNR [60] & FR & FO \\
\hline \multirow[t]{2}{*}{$1^{1} S_{0}$} & $0^{-}$ & $D^{0}$ & $1864.83 \pm 0.05$ & 1871 & 1877 & 1884 & 1874 & 1865 & 1859 \\
\hline & & $D^{ \pm}$ & $1869.58 \pm 0.09$ & & & & & & \\
\hline \multirow[t]{2}{*}{$2^{1} S_{0}$} & $0^{-}$ & $D(2550)^{0} ?$ & $2564 \pm 20$ & 2581 & 2581 & 2582 & 2540 & 2562 & \\
\hline & & $D_{J}(2580)^{0} ?$ & $2579.5 \pm 3.4 \pm 5.5[46]$ & & & & & & \\
\hline $3^{1} S_{0}$ & $0^{-}$ & $D_{J}(3000)^{0} ?$ & $2971.8 \pm 8.7[46]$ & 3062 & 3068 & 3186 & 2904 & 2970 & \\
\hline $4^{1} S_{0}$ & $0^{-}$ & & & 3452 & 3468 & 3746 & 3175 & 3279 & \\
\hline $5^{1} S_{0}$ & $0^{-}$ & & & 3793 & 3814 & 4283 & & 3535 & \\
\hline \multirow[t]{2}{*}{$1^{3} S_{1}$} & $1^{-}$ & $D^{*}(2007)^{0}$ & $2006.85 \pm 0.05$ & 2010 & 2041 & 2010 & 2006 & 2003 & 2002 \\
\hline & & $D^{*}(2010)^{ \pm}$ & $2010.26 \pm 0.05$ & & & & & & \\
\hline $2^{3} S_{1}$ & $1^{-}$ & $D_{1}^{*}(2600) ?$ & $2608.7 \pm 2.4 \pm 2.5[45]$ & 2632 & 2643 & 2655 & 2601 & 2616 & \\
\hline $3^{3} S_{1}$ & $1^{-}$ & $D_{J}^{*}(3000)^{0} ?$ & $3008.1 \pm 4.0[46]$ & 3096 & 3110 & 3239 & 2947 & 3004 & \\
\hline $4^{3} S_{1}$ & $1^{-}$ & & & 3482 & 3497 & 3789 & 3208 & 3304 & \\
\hline $5^{3} S_{1}$ & $1^{-}$ & & & 3822 & 3837 & 4319 & & 3554 & \\
\hline \multirow[t]{2}{*}{$1^{3} P_{0}$} & $0^{+}$ & $D_{0}^{*}(2400)^{0}$ & $2318 \pm 29$ & 2406 & 2399 & 2357 & 2341 & & 2319 \\
\hline & & $D_{0}^{*}(2400)^{ \pm}$ & $2351 \pm 7$ & & & & & & \\
\hline $1^{1} P_{1}$ & $1^{+}$ & $D_{1}(2420)^{0}$ & $2420.8 \pm 0.5$ & 2426 & 2456 & 2425 & 2389 & & 2411 \\
\hline \multirow[t]{2}{*}{$1^{3} P_{1}$} & $1^{+}$ & $D_{1}(2430)^{0}$ & $2427 \pm 26 \pm 25$ & 2469 & 2467 & 2447 & 2407 & & 2427 \\
\hline & & $D_{1}(2420)^{ \pm}$ & $2423.2 \pm 2.4$ & & & & & & \\
\hline \multirow[t]{2}{*}{$1^{3} P_{2}$} & $2^{+}$ & $D_{2}^{*}(2460)^{0}$ & $2460.57 \pm 0.15$ & 2460 & 2502 & 2461 & 2477 & 2461 & 2456 \\
\hline & & $D_{2}^{*}(2460)^{ \pm}$ & $2465.4 \pm 1.3$ & & & & & & \\
\hline $2^{3} P_{2}$ & $2^{+}$ & & & 3012 & 2957 & 3039 & 2860 & 2893 & \\
\hline $3^{3} P_{2}$ & $2^{+}$ & $D_{2}^{*}(3000) ?$ & $3214 \pm 29 \pm 33 \pm 36[61]$ & 3407 & 3353 & 3584 & 3142 & 3214 & \\
\hline $1^{3} D_{1}$ & $1^{-}$ & $D_{1}^{*}(2760) ?$ & $2781 \pm 18 \pm 11 \pm 6[55,56]$ & 2788 & 2817 & 2755 & 2750 & & 2775 \\
\hline $1^{1} D_{2}$ & $2^{-}$ & $D(2750)^{0} ?$ & $2752.4 \pm 1.7 \pm 2.7[45]$ & 2806 & 2816 & 2754 & 2689 & & 2789 \\
\hline $1^{3} D_{2}$ & $2^{-}$ & $D(2740)^{0} ?$ & $2737.0 \pm 3.5 \pm 11.2$ & 2850 & 2845 & 2783 & 2727 & & 2737 \\
\hline $1^{3} D_{3}$ & $3^{-}$ & $D_{3}^{*}(2760)^{0} ?$ & $2775.5 \pm 4.5 \pm 4.5 \pm 4.7[61]$ & 2863 & 2833 & 2788 & 2688 & & 2796 \\
\hline $1^{3} F_{2}$ & $2^{+}$ & & & 3090 & 3132 & & & & 3105 \\
\hline $1^{1} F_{3}$ & $3^{+}$ & & & 3129 & 3108 & & & & 3087 \\
\hline $1^{3} F_{3}$ & $3^{+}$ & & & 3145 & 3143 & & & & 2998 \\
\hline $1^{3} F_{4}$ & $4^{+}$ & & & 3187 & 3113 & & & & 3073 \\
\hline
\end{tabular}

$D_{s 1}^{*}(2700)^{ \pm}$is assigned as the $2^{3} S_{1}$ state [40,62-64]. $D_{s 1}^{*}(2700)^{ \pm}$is taken as the $2^{1} S_{0}$ state in Ref. [65] or is suggested to be a mixture of the $2^{3} S_{1}$ and $1^{3} D_{1}$ states [66-68]. In Refs. [57,69-71], $D_{s 1}^{*}(2860)$ and $D_{s 3}^{*}(2860)$ are taken as the $1^{3} D_{1}$ state and the $1^{3} D_{3}$ state, respectively. In Ref. [72], $D_{s 1}^{*}(2860)$ is interpreted as a mixture of $D_{s}\left(2^{3} S_{1}\right)$ and $D_{s}\left(1^{3} D_{1}\right)$.

\section{$3.4 B / B_{S} / B_{C}$}

For the bottom mesons, the bottom-strange meson, especially for the bottom-charmed mesons, the experimental data are scarce and the theoretical values are used to fit the radial and orbital Regge trajectories.
For the bottom mesons, the theoretical values in [60] are used. The fitted universal parameters are $\beta=2.69$ and $c_{l}=$ 1.74. The Regge trajectories are shown in Fig. 5, and the experimental and theoretical values are listed in Table 4.

$B_{J}(5840)^{0}$ is suggested to be the $2^{1} S_{0}$ state $[73,74]$. The $B_{J}(5960)^{0}$ and $B(5970)^{0}$ are probably the same state. In [74], $B(5970)^{0} / B_{J}(5960)^{0}$ is interpreted as $2^{3} S_{1}$ or $1^{3} D_{3}$ states [75]. In Refs. [76,77], $B(5970)^{0}$ is takes as the $2^{3} S_{1}$ state. In Ref. [78], $B(5970)^{0}$ is assumed to be the $2^{3} S_{1}, 1^{3} D_{1}$ and $1^{3} D_{3}$ states.

For the bottom-strange mesons, $\beta=1.75$ and $c_{l}=2.44$. The radial and orbital Regge trajectories are given by fitting the theoretical predictions in Ref. [60], see Fig. 6. The experimental and theoretical data are in Table 5. 


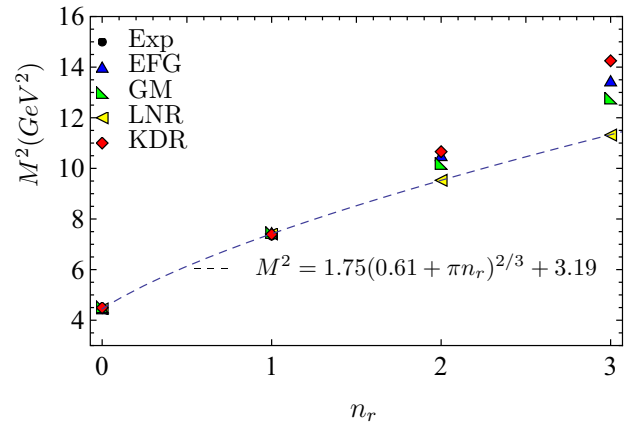

(a)

Fig. 4 (Color online) The radial and orbital Regge trajectories for the charmed-strange mesons. The theoretical data are from Ref. [16] (EFG), [53] (GM), [54] (KDR) and [60] (LNR). The experimental data (Exp)

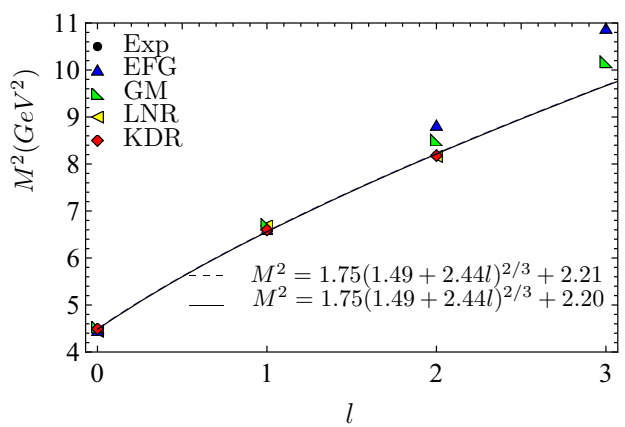

(b)

are from Ref. [39]. The dashed lines are the fitted Regge trajectories for the theoretical values in [60] while the solid line is for the experimental data from [39]

Table 3 Masses of the charmed-strange mesons (in MeV). The experimental data are from PDG [39]. The theoretical values are from EFG [16], GM [53], KDR [54] and LNR [60]. ? denotes the possible candidates for the unwell-established states or the mesons needing confirmation

\begin{tabular}{llllllll}
\hline State & $J^{P}$ & Meson & PDG [39] & EFG [16] & GM [53] & KDR [54] & LNR [60] \\
\hline $1^{1} S_{0}$ & $0^{-}$ & $D_{s}^{ \pm}$ & $1968.27 \pm 0.10$ & 1969 & 1979 & 1965 & 1975 \\
$1^{3} S_{1}$ & $1^{-}$ & $D_{s}^{* \pm}$ & $2112.1 \pm 0.4$ & 2111 & 2129 & 2120 & 2108 \\
$2^{3} S_{1}$ & $1^{-}$ & $D_{s 1}^{*}(2700)^{ \pm} ?$ & $2708.3_{-3.4}^{+4.0}$ & 2731 & 2732 & 2719 & 2722 \\
$3^{3} S_{1}$ & $1^{-}$ & & & 3242 & 3193 & 3265 & 3087 \\
$4^{3} S_{1}$ & $1^{-}$ & & & 3669 & 3575 & 3775 & 3364 \\
$1^{3} P_{0}$ & $0^{+}$ & $D_{s 0}^{*}(2317)^{ \pm}$ & $2317.7 \pm 0.6$ & 2509 & 2484 & 2438 & 2455 \\
$1^{1} P_{1}$ & $1^{+}$ & $D_{s 1}(2536)^{ \pm}$ & $2535.10 \pm 0.06$ & 2536 & 2549 & 2529 & 2502 \\
$1^{3} P_{1}$ & $1^{+}$ & $D_{s 1}(2460)^{ \pm}$ & $2459.5 \pm 0.6$ & 2574 & 2556 & 2541 & 2522 \\
$1^{3} P_{2}$ & $2^{+}$ & $D_{s 2}^{*}(2573)$ & $2569.1 \pm 0.8$ & 2571 & 2592 & 2569 & 2586 \\
$1^{3} D_{1}$ & $1^{-}$ & $D_{s 1}^{*}(2860)^{ \pm} ?$ & $2859 \pm 12 \pm 24$ & 2913 & 2899 & 2882 & 2845 \\
$1^{1} D_{2}$ & $2^{-}$ & & & 2931 & 2900 & 2853 & 2838 \\
$1^{3} D_{3}$ & $3^{-}$ & $D_{s 3}^{*}(2860)^{ \pm} ?$ & $2860.5 \pm 2.6 \pm 6.5$ & 2971 & 2917 & 2860 & 2857 \\
$1^{3} F_{2}$ & $2^{+}$ & & 3230 & 3208 & 3186 & 3190 & \\
$1^{1} F_{3}$ & $3^{+}$ & & & 3254 & 3300 & & \\
$1^{3} F_{4}$ & $4^{+}$ & & & & &
\end{tabular}

By fitting the theoretical values in Ref. [79], we obtain the Regge trajectories for the bottom-charmed mesons, $\beta=3.49$ and $c_{l}=1.75$, see Fig. 7. In Table 6 are the experimental and theoretical values.

Up to date, only two $B_{c}$ states have been observed. $B_{c}^{+}$ is well established [80-84]. $B_{c}(2 S)$ was observed by the ATLAS Collaboration [85] but has not been confirmed [86].

\section{Discussions}

In this section, two important properties, the universality and the concavity, of the Regge trajectories are discussed.
4.1 Universality of the new form of the Regge trajectories

In Ref. [25], we obtained one new form of the Regge trajectories [Eq. (1)] from the QSSE and showed that heavy quarkonia can be well described by Eq. (1) by fitting the spectra of charmonia and bottomonia. In the present work, the case of the mesons consisting of unequally massive quarks are considered and the obtained Regge trajectories have the same form as the Regge trajectories for heavy quarkonia. Then we apply the obtained Regge trajectories to fit the spectra of the strange mesons, the heavy-light mesons (the $D, D_{s}, B$ and $B_{s}$ mesons) and the $B_{c}$ mesons. The fitted Regge trajectories are in agreement with the experimental data and the theoretical predictions. The new form of the Regge trajectories is expected to be appropriate also for the light unflavored 


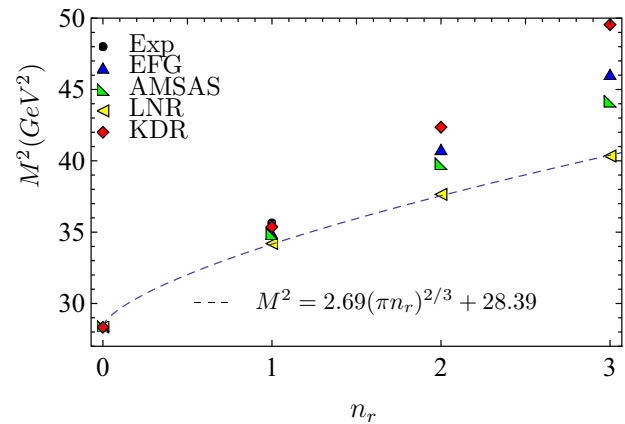

(a)

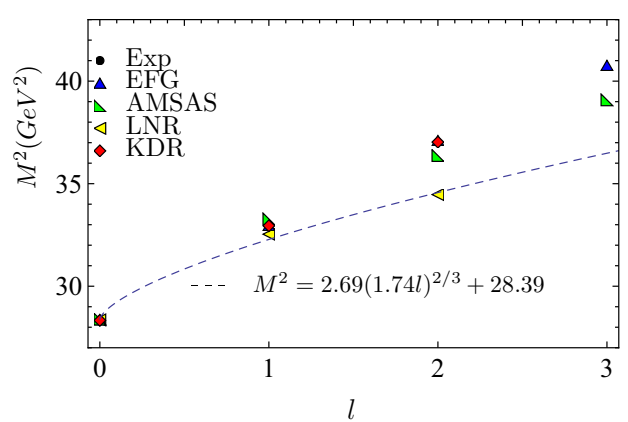

(b)

Fig. 5 (Color online) The radial and orbital Regge trajectories for the bottom mesons. The theoretical data are from Ref. [16] (EFG), [88] (AMSAS), [87] (KDR) and [60] (LNR). The experimental data (Exp) are from Ref. [39]. The dashed lines are the Regge trajectories by fitting the theoretical values in [60]

Table 4 Masses of the bottom mesons (in MeV). The experimental data are from PDG [39]. The theoretical values are from EFG [16],KDR [87], LNR [60] and AMSAS [88]. ? denotes the possible candidates for the unwell-established states or the mesons needing confirmation

\begin{tabular}{|c|c|c|c|c|c|c|c|}
\hline State & $J^{P}$ & Meson & PDG [39] & EFG [16] & KDR [87] & LNR [60] & AMSAS [88] \\
\hline $1^{1} S_{0}$ & $0^{-}$ & $B^{0}$ & $5279.62 \pm 0.15$ & 5280 & 5287 & 5277 & 5268 \\
\hline $2^{1} S_{0}$ & $0^{-}$ & $B_{J}(5840)^{0} ?$ & $5863 \pm 9$ & 5890 & 5926 & 5822 & 5877 \\
\hline $3^{1} S_{0}$ & $0^{-}$ & & & 6379 & 6492 & 6117 & 6288 \\
\hline $4{ }^{1} S_{0}$ & $0^{-}$ & & & 6781 & 7027 & 6335 & 6631 \\
\hline $1^{3} S_{1}$ & $1^{-}$ & $B^{*}$ & $5324.65 \pm 0.25$ & 5326 & 5323 & 5325 & 5329 \\
\hline $2^{3} S_{1}$ & $1^{-}$ & $B_{J}(5960)^{0} ?$ & $5969.2 \pm 2.9 \pm 5.1 \pm 0.2[73]$ & 5906 & 5947 & 5848 & 5905 \\
\hline $3^{3} S_{1}$ & $1^{-}$ & & & 6387 & 6508 & 6136 & 6308 \\
\hline $4^{3} S_{1}$ & $1^{-}$ & & & 6786 & 7039 & 6351 & 6647 \\
\hline $1^{1} P_{1}$ & $1^{+}$ & $B_{1}(5721)^{0}$ & $5726.0 \pm 1.3$ & 5723 & 5733 & 5686 & 5755 \\
\hline $1^{3} P_{2}$ & $2^{+}$ & $B_{2}^{*}(5747)^{0}$ & $5739.5 \pm 0.7$ & 5741 & 5740 & 5704 & 5769 \\
\hline $1^{3} D_{3}$ & $3^{-}$ & & & 6091 & 6085 & 5871 & 6031 \\
\hline $1^{3} F_{4}$ & $4^{+}$ & & & 6385 & & & 6252 \\
\hline
\end{tabular}

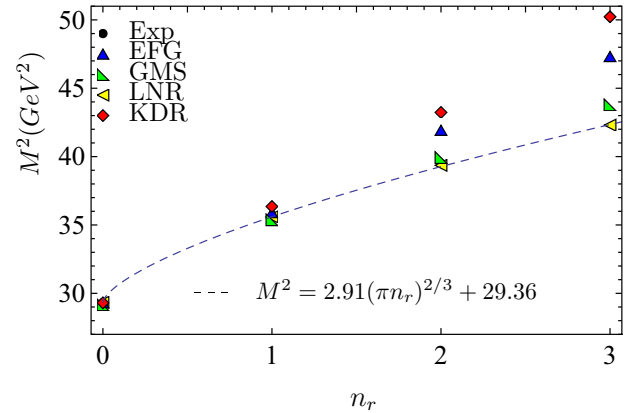

(a)

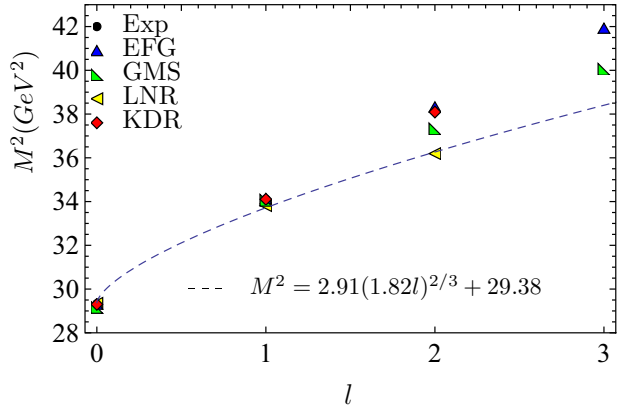

(b)

Fig. 6 (Color online) The radial and orbital Regge trajectories for the bottom-strange mesons. The theoretical data are from Ref. [16] (EFG), [89] (GMS), [87] (KDR) and [60] (LNR). The experimental data (Exp) are from Ref. [39]. The dashed lines are the Regge trajectories by fitting the theoretical values in [60]

mesons, for example, see Fig. 8. Therefore, we conclude that the new form the Regge trajectories [Eq. (1)] can be appropriate not only for the heavy mesons but also for the light mesons, not only for the mesons composed of the same quarks but also for the mesons composed of different quarks, that is to say, Eq. (1) is expected to be universal for mesons.

The parameters $\beta_{n_{r}}$ of the radial Regge trajectories and $\beta_{l}$ of the orbital Regge trajectories are listed in Tables 7 and 8 , respectively. For mesons except for the light mesons, $\beta_{n_{r}}$ 
Table 5 Masses of the bottom mesons (in MeV). The experimental data are from PDG [39]. The theoretical values are from EFG [16], GMS [89], LNR [60] and KDR [87]. ? denotes the possible candidates for the unwell-established states or the mesons needing confirmation

\begin{tabular}{|c|c|c|c|c|c|c|c|}
\hline State & $J^{P}$ & Meson & PDG [39] & EFG [16] & GMS [89] & LNR [60] & KDR [87] \\
\hline $1^{1} S_{0}$ & $0^{-}$ & $B_{s}^{0}$ & $5366.82 \pm 0.22$ & 5372 & 5366 & 5366 & 5367 \\
\hline $1^{3} S_{1}$ & $1^{-}$ & $B_{s}^{*}$ & $5415.4_{-1.5}^{+1.8}$ & 5414 & 5400 & 5417 & 5413 \\
\hline $2^{3} S_{1}$ & $1^{-}$ & & & 5992 & 5948 & 5966 & 6029 \\
\hline $3^{3} S_{1}$ & $1^{-}$ & & & 6475 & 6319 & 6274 & 6575 \\
\hline $4^{3} S_{1}$ & $1^{-}$ & & & 6879 & 6617 & 6504 & 7087 \\
\hline $1^{1} P_{1}$ & $1^{+}$ & $B_{s 1}(5830)^{0}$ & $5828.63 \pm 0.27$ & 5831 & 5830 & 5795 & 5842 \\
\hline $1^{3} P_{2}$ & $2^{+}$ & $B_{s 2}^{*}(5840)^{0}$ & $5839.84 \pm 0.18$ & 5842 & 5836 & 5815 & 5840 \\
\hline $1^{3} D_{3}$ & $3^{-}$ & & & 6191 & 6109 & 6016 & 6172 \\
\hline $1^{3} F_{4}$ & $4^{+}$ & & & 6475 & 6328 & & \\
\hline
\end{tabular}

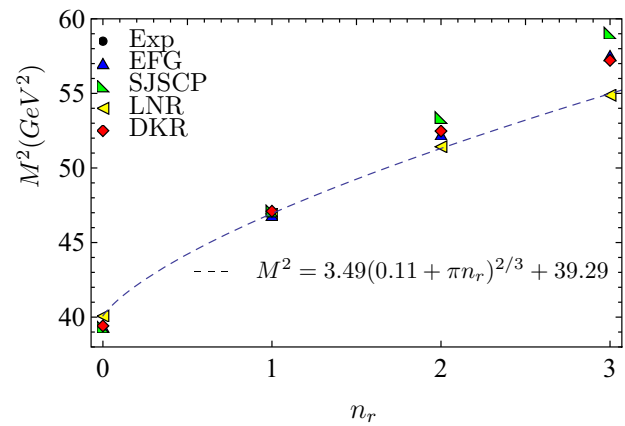

(a)

Fig. 7 (Color online) The radial and orbital Regge trajectories for the bottom-charmed mesons. The theoretical data are from Ref. [90] (EFG), [91] (SJSCP), [92] (DKR) and [79] (LNR). The experimental data (Exp)

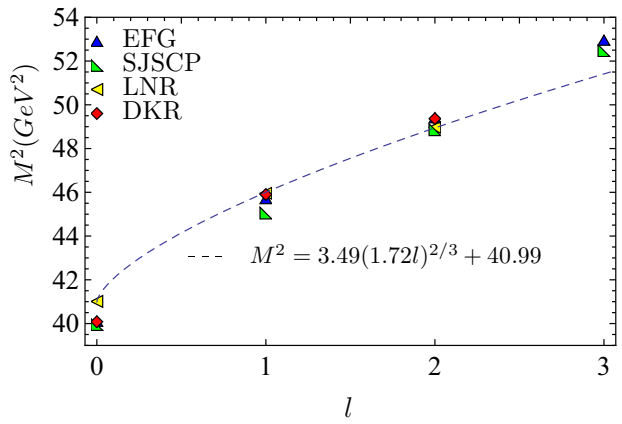

(b)

are from Ref. [39]. The dashed lines are the Regge trajectories by fitting the theoretical values in [79]

Table 6 Masses of the bottom mesons (in MeV). The experimental data are from PDG [39]. The theoretical values are from EFG [16],KDR [87], LNR [60] and AMSAS [88]. ? denotes the possible candidates for the unwell-established states or the mesons needing confirmation

\begin{tabular}{|c|c|c|c|c|c|c|c|}
\hline State & $J^{P}$ & Meson & PDG [39] & EFG [90] & SJSCP [91] & DKR [92] & LNR [79] \\
\hline $1^{1} S_{0}$ & $0^{-}$ & $B_{c}^{+}$ & $6275.1 \pm 1.0$ & 6272 & 6272 & 6278 & 6330 \\
\hline $2^{1} S_{0}$ & $0^{-}$ & $B_{c}(2 S)^{ \pm} ?$ & $6842 \pm 4 \pm 5$ & 6842 & 6864 & 6863 & 6850 \\
\hline $3^{1} S_{0}$ & $0^{-}$ & & & 7226 & 7306 & 7244 & 7171 \\
\hline $4^{1} S_{0}$ & $0^{-}$ & & & 7585 & 7684 & 7564 & 7408 \\
\hline $1^{3} S_{1}$ & $1^{-}$ & & & 6333 & 6321 & 6331 & 6404 \\
\hline $1^{3} P_{2}$ & $2^{+}$ & & & 6761 & 6712 & 6775 & 6779 \\
\hline $1^{3} D_{3}$ & $3^{-}$ & & & 7029 & 6990 & 7026 & 6998 \\
\hline $1^{3} F_{4}$ & $4^{+}$ & & & 7277 & 7244 & & \\
\hline
\end{tabular}

and $\beta_{l}$ increase with the quark mass, and there is the relation $\beta_{n_{r}}>\beta_{l}$. The exception of the light mesons maybe arises from the complexity of the light mesons.

\subsection{Concavity of the Regge trajectories}

In Refs. [23,90,93-98], the fitted curves showed evidently the concavity of the Regge trajectories although the authors did not point out explicitly the curvature of the meson Regge trajectories. In Refs. [14,99, 100], the authors noticed the curvature of the Regge trajectories for mesons but a few Regge trajectories were fitted, therefore, the generality of the concavity can not be illustrated.

In Ref. [25], we have shown that the bottomonia and charmonia can be well described by the concave formula (1). In Sect. 3, the strange mesons, the heavy-light mesons (the 


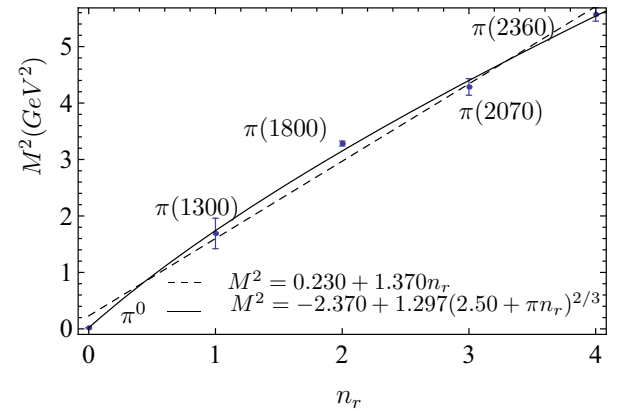

Fig. 8 The radial Regge trajectories for $\pi$ [25]

Table $7 \beta_{n_{r}}$ of the radial Regge trajectories. $\beta_{n_{r}}=\beta \pi^{2 / 3} . n$ represents the up quark or down quark

\begin{tabular}{lllll}
\hline & $\bar{n}$ & $\bar{s}$ & $\bar{c}$ & $\bar{b}$ \\
\hline$n$ & $2.78^{\mathrm{a}}$ & 2.06 & 4.05 & $5.77^{\mathrm{c}}$ \\
$s$ & 2.06 & $-^{\mathrm{b}}$ & $3.75^{\mathrm{c}}$ & $6.24^{\mathrm{c}}$ \\
$c$ & 4.05 & $3.75^{\mathrm{c}}$ & 4.33 & $7.49^{\mathrm{c}}$ \\
$b$ & $5.77^{\mathrm{c}}$ & $6.24^{\mathrm{c}}$ & $7.49^{\mathrm{c}}$ & 10.94
\end{tabular}

${ }^{\mathrm{a}}$ The coefficient is from the radial Regge trajectory for $\pi^{0}$

${ }^{\mathrm{b}}$ The radial Regge trajectories for $\eta^{\prime}(958)$ and for $\phi(1020)$ seem convex which need confirmation. See Ref. [101] for more discussions

${ }^{\mathrm{c}}$ These coefficients are from the radial Regge trajectories by fitting the theoretical values in Refs. $[60,79]$

Table $8 \beta_{l}$ of the orbital Regge trajectories. $\beta_{l}=\beta c_{l}^{2 / 3} . n$ represents the up quark or down quark

\begin{tabular}{lllll}
\hline & $\bar{n}$ & $\bar{s}$ & $\bar{c}$ & $\bar{b}$ \\
\hline$n$ & $3.89^{\mathrm{a}}$ & 2.61 & 3.95 & $3.89^{\mathrm{c}}$ \\
$s$ & 2.61 & $2.36^{\mathrm{b}}$ & $3.17^{\mathrm{c}}$ & $4.34^{\mathrm{c}}$ \\
$c$ & 3.95 & $3.17^{\mathrm{c}}$ & 3.53 & $5.01^{\mathrm{c}}$ \\
$b$ & $3.89^{\mathrm{c}}$ & $4.34^{\mathrm{c}}$ & $5.01^{\mathrm{c}}$ & 9.27 \\
\hline
\end{tabular}

${ }^{\mathrm{a}}$ The coefficient is from the orbital Regge trajectory for $\rho(770)$

${ }^{\mathrm{b}}$ The coefficient is from the orbital Regge trajectory for $\phi(1020)$

${ }^{c}$ These coefficients are from the orbital Regge trajectories by fitting the theoretical values in Refs. [60,79]

$D, D_{s}, B$ and $B_{s}$ ) and the bottom-charmed mesons are discussed by the formula (1). The Regge trajectories [Eq. (1)] are appropriate also for some light unflavored mesons, for example, see Fig. 8. The Regge trajectories will be concave if the spectra can be described by a concave formula, therefore, the concavity of the Regge trajectories for these mesons is independent of the models which the employed formula comes from. See Ref. [101] for more discussions.

It is one of the fundamental properties whether the meson Regge trajectories are linear, concave, convex or have the inflection points. For the potential models, the concavity of the Regge trajectories is of cardinal significance because it is related to the choice of the appropriate dynamic equation or the appropriate potential. If the confining potential is lin- ear [102-116], the Schrödinger equation produces the convex Regge trajectories while the spinless Salpeter equation [13,117], the Dirac equation [118] and the Klein-Gordon equation [119-123] give the linear Regge trajectories. The QSSE $[25,31,101]$ and the eigenvalue equation for the square mass operator lead to the concave Regge trajectories. There exist other possibilities. For example, if the confining potential takes the form $r^{0.1}[124,125]$ or $r^{1 / 2}$ [126-128], all dynamic equations mentioned above can yield the concave Regge trajectories. In this case, more information is needed to choose the appropriate dynamic equation and the corresponding confinement potential.

\section{Conclusions}

In this paper, we obtain the radial and orbital Regge trajectories for the mesons composed of unequally massive quarks from the QSSE by employing the Bohr-Sommerfeld quantization approach. The obtained Regge trajectories have the same form as the Regge trajectories for heavy quarkonia [25], $M^{2}=\beta\left(c_{l} l+\pi n_{r}+c_{0}\right)^{2 / 3}+c_{1}$. By fitting the spectra of the strange mesons, the heavy-light mesons (the $D, D_{s}, B$ and $B_{s}$ mesons) and the bottom-charmed mesons, we show that the fitted Regge trajectories are in agreement with the experimental data and the theoretical predictions. By combining the results in Ref. [25] and in the present work, we expect that the new form of the Regge trajectories will be universal for mesons.

By fitting the spectra of mesons, the concavity of these Regge trajectories is illustrated. This property is of cardinal significance for the potential models because it can assist in the choice of the appropriate dynamic equation or the appropriate potential to describe mesons. If the confinement potential is linear, the Schrödinger equation arouses the convex Regge trajectories while the Dirac equation, the KleinGordon equation and the spinless Salpeter equation will produce the linear Regge trajectories. The QSSE [Eq. (4)] and the eigenvalue equation for the square mass operator [Eq. (2)] can give the concave Regge trajectories. Therefore, the QSSE and the eigenvalue equation for the square mass operator are preferred if the confinement potential is linear. If the confinement potential takes the form $r^{0.1}$ or $r^{1 / 2}$, all the dynamic equations mentioned above can yield the concave Regge trajectories. In this case, more information is needed to choose the appropriate dynamic equation and the corresponding potential.

Open Access This article is distributed under the terms of the Creative Commons Attribution 4.0 International License (http://creativecomm ons.org/licenses/by/4.0/), which permits unrestricted use, distribution, and reproduction in any medium, provided you give appropriate credit to the original author(s) and the source, provide a link to the Creative Commons license, and indicate if changes were made. Funded by SCOAP $^{3}$. 


\section{References}

1. T. Regge, Nuovo Cim. 14, 951 (1959)

2. G.F. Chew, S.C. Frautschi, Phys. Rev. Lett. 7, 394 (1961)

3. G.F. Chew, S.C. Frautschi, Phys. Rev. Lett. 8, 41 (1962)

4. Y. Nambu, Phys. Rev. D 10, 4262 (1974)

5. Y. Nambu, Phys. Lett. B 80, 372 (1979)

6. M. Ademollo, G. Veneziano, S. Weinberg, Phys. Rev. Lett. 22, 83 (1969)

7. M. Baker, R. Steinke, Phys. Rev. D 65, 094042 (2002). arXiv:hep-th/0201169

8. J. Polchinski, M.J. Strassler, Phys. Rev. Lett. 88, 031601 (2002). arXiv:hep-th/0109174

9. A. Karch, E. Katz, D.T. Son, M.A. Stephanov, Phys. Rev. D 74, 015005 (2006). arXiv:hep-ph/0602229

10. S.J. Brodsky, Eur. Phys. J. A 31, 638 (2007). arXiv:hep-ph/0610115

11. H. Forkel, M. Beyer, T. Frederico, JHEP 0707, 077 (2007). arXiv:0705.1857 [hep-ph]

12. S. Filipponi, Y. Srivastava, Phys. Rev. D 58, 016003 (1998). arXiv:hep-ph/9712204

13. F. Brau, Phys. Rev. D 62, 014005 (2000). arXiv:hep-ph/0412170

14. M.M. Brisudova, L. Burakovsky, T. Goldman, Phys. Rev. D 61, 054013 (2000). arXiv:hep-ph/9906293

15. X.H. Guo, K.W. Wei, X.H. Wu, Phys. Rev. D 78, 056005 (2008). arXiv:0809.1702 [hep-ph]

16. D. Ebert, R.N. Faustov, V.O. Galkin, Eur. Phys. J. C 66, 197 (2010). arXiv:0910.5612 [hep-ph]

17. D. Ebert, R.N. Faustov, V.O. Galkin, Phys. Rev. D 79, 114029 (2009). arXiv:0903.5183 [hep-ph]

18. W. Buchmuller, G. Grunberg, S.H.H. Tye, Phys. Rev. Lett. 45, 103 (1980) [Erratum: Phys. Rev. Lett. 45, 587 (1980)]

19. J.L. Gervais, A. Neveu, Nucl. Phys. B 163, 189 (1980)

20. C. Lovelace, Phys. Lett. 28B, 264 (1968)

21. A.C. Irving, R.P. Worden, Phys. Rep. 34, 117 (1977)

22. P.D.B. Collins, Phys. Rep. 1, 103 (1971)

23. G.S. Sharov, arXiv:hep-ph/1305.3985

24. K. Chen, Y.B. Dong, X. Liu, Q.F. Lü, T. Matsuki, Eur. Phys. J. C 78(1), 20 (2018). arXiv:1709.07196 [hep-ph]

25. J.K. Chen, Eur. Phys. J. C 78(3), 235 (2018)

26. M. Baldicchi, Conference: C99-09-29, 325 (2001). arXiv:hep-ph/9911268

27. N. Brambilla, E. Montaldi, G.M. Prosperi, Phys. Rev. D 54, 3506 (1996). arXiv:hep-ph/9504229

28. M. Baldicchi, A.V. Nesterenko, G.M. Prosperi, C. Simolo, Phys. Rev. D 77, 034013 (2008). arXiv:0705.1695 [hep-ph]

29. M. Baldicchi, A.V. Nesterenko, G.M. Prosperi, D.V. Shirkov, C. Simolo, Phys. Rev. Lett. 99, 242001 (2007). arXiv:0705.0329 [hep-ph]

30. M. Baldicchi, G.M. Prosperi, Phys. Rev. D 62, 114024 (2000). arXiv:hep-ph/0008017

31. E. Di Salvo, L. Kondratyuk, P. Saracco, Z. Phys. C 69, 149 (1995). arXiv:hep-ph/9411309

32. J.K. Chen, Acta Phys. Pol. B 47, 1155 (2016)

33. J.K. Chen, Rom. J. Phys. 62, 119 (2017)

34. S. Tomonaga, Quantum Mechanics, Volume I: Old Quantum Theory (North-Holland Publishing Company, Amsterdam, 1962)

35. E.E. Salpeter, H.A. Bethe, Phys. Rev. 84, 1232 (1951)

36. E.E. Salpeter, Phys. Rev. 87, 328 (1952)

37. E. Eichten, K. Gottfried, T. Kinoshita, J.B. Kogut, K.D. Lane, T.M. Yan, Phys. Rev. Lett. 34, 369 (1975) [Erratum: Phys. Rev. Lett. 36, 1276 (1976)]

38. I.S. Gradshteyn, I.M. Ryzhik, Table of Integrals, Series, and Prod$u c t s$, corrected and enlarged edn. (Academic Press, New York, 1980)
39. C. Patrignani et al. (Particle Data Group), Chin. Phys. C 40(10), $100001(2016)$

40. S. Godfrey, N. Isgur, Phys. Rev. D 32, 189 (1985)

41. C.Q. Pang, J.Z. Wang, X. Liu, T. Matsuki, Eur. Phys. J. C 77(12), 861 (2017). arXiv:1705.03144 [hep-ph]

42. R. Aaij et al. (LHCb Collaboration), Phys. Rev. Lett. 118(2), 022003 (2017). arXiv:1606.07895 [hep-ex]

43. T. Barnes, N. Black, P.R. Page, Phys. Rev. D 68, 054014 (2003). arXiv:nucl-th/0208072

44. J. Vijande, F. Fernandez, A. Valcarce, J. Phys. G 31, 481 (2005). arXiv:hep-ph/0411299

45. P. del Amo Sanchez et al. (BaBar Collaboration), Phys. Rev. D 82, 111101 (2010). arXiv:1009.2076 [hep-ex]

46. R. Aaij et al. (LHCb Collaboration), JHEP 1309, 145 (2013). arXiv:1307.4556 [hep-ex]

47. Y. Sun, X. Liu, T. Matsuki, Phys. Rev. D 88(9), 094020 (2013). arXiv:1309.2203 [hep-ph]

48. Q.F. Lü, D.M. Li, Phys. Rev. D 90(5), 054024 (2014). arXiv:1407.3092 [hep-ph]

49. G.L. Yu, Z.G. Wang, Z.Y. Li, G.Q. Meng, Chin. Phys. C 39(6), 063101 (2015). arXiv:1402.5955 [hep-ph]

50. Z.G. Wang, Phys. Rev. D 83, 014009 (2011). arXiv:1009.3605 [hep-ph]

51. H.X. Chen, W. Chen, X. Liu, Y.R. Liu, S.L. Zhu, Rep. Prog. Phys. 80(7), 076201 (2017). arXiv:1609.08928 [hep-ph]

52. J.Z. Wang, D.Y. Chen, Q.T. Song, X. Liu, T. Matsuki, Phys. Rev. D 94(9), 094044 (2016). arXiv:1608.04186 [hep-ph]

53. S. Godfrey, K. Moats, Phys. Rev. D 93(3), 034035 (2016). arXiv: 1510.08305 [hep-ph]

54. V. Kher, N. Devlani, A.K. Rai, Chin. Phys. C 41(7), 073101 (2017). arXiv:1704.00439 [hep-ph]

55. R. Aaij et al. (LHCb Collaboration), Phys. Rev. D 91(9), 092002 (2015)

56. R. Aaij et al. (LHCb Collaboration), Phys. Rev. D 93(11), 119901(E) (2016). arXiv:1503.02995 [hep-ex]

57. J.B. Liu, C.D. Lü, Eur. Phys. J. C 77(5), 312 (2017). arXiv:1605.05550 [hep-ph]

58. R. Aaij et al. (LHCb Collaboration), Phys. Rev. D 92(3), 032002 (2015). arXiv:1505.01710 [hep-ex]

59. B. Chen, X. Liu, A. Zhang, Phys. Rev. D 92(3), 034005 (2015). arXiv: 1507.02339 [hep-ph]

60. T.A. Lahde, C.J. Nyfalt, D.O. Riska, Nucl. Phys. A 674, 141 (2000). arXiv:hep-ph/9908485

61. R. Aaij et al. (LHCb Collaboration), Phys. Rev. D 94(7), 072001 (2016). arXiv:1608.01289 [hep-ex]

62. B. Zhang, X. Liu, W.Z. Deng, S.L. Zhu, Eur. Phys. J. C 50, 617 (2007). arXiv:hep-ph/0609013

63. J. Segovia, D.R. Entem, F. Fernandez, Phys. Rev. D 91(9), 094020 (2015). arXiv:1502.03827 [hep-ph]

64. G.L. Wang, J.M. Zhang, Z.H. Wang, Phys. Lett. B 681, 326 (2009). arXiv:1001.2035 [hep-ph]

65. P. Colangelo, F. De Fazio, S. Nicotri, M. Rizzi, Phys. Rev. D 77, 014012 (2008). arXiv:0710.3068 [hep-ph]

66. F.E. Close, C.E. Thomas, O. Lakhina, E.S. Swanson, Phys. Lett. B 647, 159 (2007). arXiv:hep-ph/0608139

67. D.M. Li, B. Ma, Phys. Rev. D 81, 014021 (2010). arXiv:0911.2906 [hep-ph]

68. X.H. Zhong, Q. Zhao, Phys. Rev. D 81, 014031 (2010). arXiv:0911.1856 [hep-ph]

69. Q.T. Song, D.Y. Chen, X. Liu, T. Matsuki, Eur. Phys. J. C 75(1), 30 (2015). arXiv:1408.0471 [hep-ph]

70. S. Godfrey, K. Moats, Phys. Rev. D 90(11), 117501 (2014). arXiv: 1409.0874 [hep-ph]

71. Z.G. Wang, Eur. Phys. J. C 75(1), 25 (2015). arXiv:1408.6465 [hep-ph] 
72. Q.T. Song, D.Y. Chen, X. Liu, T. Matsuki, Phys. Rev. D 91, 054031 (2015). arXiv:1501.03575 [hep-ph]

73. R. Aaij et al. (LHCb Collaboration), JHEP 1504, 024 (2015). arXiv:1502.02638 [hep-ex]

74. Q.F. Lü, T.T. Pan, Y.Y. Wang, E. Wang, D.M. Li, Phys. Rev. D 94(7), 074012 (2016). arXiv:1607.02812 [hep-ph]

75. L.Y. Xiao, X.H. Zhong, Phys. Rev. D 90(7), 074029 (2014). arXiv: 1407.7408 [hep-ph]

76. Y. Sun, Q.T. Song, D.Y. Chen, X. Liu, S.L. Zhu, Phys. Rev. D 89(5), 054026 (2014). arXiv:1401.1595 [hep-ph]

77. H. Xu, X. Liu, T. Matsuki, Phys. Rev. D 89(9), 097502 (2014). arXiv: 1402.0384 [hep-ph]

78. Z.G. Wang, Eur. Phys. J. Plus 129, 186 (2014). arXiv:1401.7580 [hep-ph]

79. T.A. Lahde, C.J. Nyfalt, D.O. Riska, Nucl. Phys. A 645, 587 (1999). arXiv:hep-ph/9808438 [Erratum: Nucl. Phys. A 665, 447 (2000)]

80. F. Abe et al. (CDF Collaboration), Phys. Rev. Lett. 81, 2432 (1998). arXiv:hep-ex/9805034

81. A. Abulencia et al. (CDF Collaboration), Phys. Rev. Lett. 96, 082002 (2006). arXiv:hep-ex/0505076

82. T. Aaltonen et al. (CDF Collaboration), Phys. Rev. Lett. 100, 182002 (2008). arXiv:0712.1506 [hep-ex]

83. V.M. Abazov et al. (D0 Collaboration), Phys. Rev. Lett. 101, 012001 (2008). arXiv:0802.4258 [hep-ex]

84. R. Aaij et al. (LHCb Collaboration), Phys. Rev. Lett. 109, 232001 (2012). arXiv:1209.5634 [hep-ex]

85. G. Aad et al. (ATLAS Collaboration), Phys. Rev. Lett. 113(21), 212004 (2014). arXiv:1407.1032 [hep-ex]

86. R. Aaij et al. (LHCb Collaboration), JHEP 1801, 138 (2018). arXiv:1712.04094 [hep-ex]

87. V. Kher, N. Devlani, A.K. Rai, Chin. Phys. C 41(9), 093101 (2017). arXiv:1705.08248 [hep-ph]

88. I. Asghar, B. Masud, E.S. Swanson, F. Akram, M. Atif Sultan. Eur. Phys. J. A 54(7), 127 (2018). arXiv:1804.08802 [hep-ph]

89. S. Godfrey, K. Moats, E.S. Swanson, Phys. Rev. D 94(5), 054025 (2016). arXiv:1607.02169 [hep-ph]

90. D. Ebert, R.N. Faustov, V.O. Galkin, Eur. Phys. J. C 71, 1825 (2011). arXiv:1111.0454 [hep-ph]

91. N.R. Soni, B.R. Joshi, R.P. Shah, H.R. Chauhan, J.N. Pandya, Eur. Phys. J. C 78(7), 592 (2018). arXiv:1707.07144 [hep-ph]

92. N. Devlani, V. Kher, A.K. Rai, Eur. Phys. J. A 50(10), 154 (2014)

93. J. Sonnenschein, D. Weissman, JHEP 1408, 013 (2014). arXiv: 1402.5603 [hep-ph]

94. S.S. Afonin, I.V. Pusenkov, Mod. Phys. Lett. A 29(35), 1450193 (2014). arXiv:1308.6540 [hep-ph]

95. T. Branz, T. Gutsche, V.E. Lyubovitskij, I. Schmidt, A. Vega, Phys. Rev. D 82, 074022 (2010). arXiv:1008.0268 [hep-ph]

96. K.W. Wei, X.H. Guo, Phys. Rev. D 81, 076005 (2010)

97. S.S. Gershtein, A.K. Likhoded, A.V. Luchinsky, Phys. Rev. D 74, 016002 (2006). arXiv:hep-ph/0602048
98. S.S. Afonin, I.V. Pusenkov, Phys. Rev. D 90(9), 094020 (2014). arXiv:1411.2390 [hep-ph]

99. A. Inopin, G.S. Sharov, Phys. Rev. D 63, 054023 (2001). arXiv:hep-ph/9905499

100. M.M. Brisudova, L. Burakovsky, J.T. Goldman, Phys. Lett. B 460, 1 (1999). arXiv:hep-ph/9810296

101. J. K. Chen, arXiv:1807.11003 [hep-ph]

102. G.S. Bali, Phys. Rep. 343, 1 (2001). arXiv:hep-ph/0001312

103. N. Brambilla et al. (Quarkonium Working Group), arXiv:hep-ph/0412158

104. T. Kawanai, S. Sasaki, Phys. Rev. D 85, 091503 (2012). arXiv: 1110.0888 [hep-lat]

105. J.B. Kogut, L. Susskind, Phys. Rev. D 9, 3501 (1974)

106. K.G. Wilson, Phys. Rev. D 10, 2445 (1974)

107. E.P. Tryon, Phys. Rev. Lett. 28, 1605 (1972)

108. L.P. Fulcher, Phys. Rev. D 50, 447 (1994)

109. C.R. Munz, J. Resag, B.C. Metsch, H.R. Petry, Nucl. Phys. A 578, 418 (1994). arXiv:nucl-th/9307027

110. R. Ricken, M. Koll, D. Merten, B.C. Metsch, Eur. Phys. J. A 18, 667 (2003). arXiv:hep-ph/0302124

111. D.P. Stanley, D. Robson, Phys. Rev. D 21, 3180 (1980)

112. E. Eichten, K. Gottfried, T. Kinoshita, K.D. Lane, T.M. Yan, Phys. Rev. D 17, 3090 (1978)

113. E. Eichten, K. Gottfried, T. Kinoshita, K.D. Lane, T.M. Yan, Phys. Rev. D 21, 313(E) (1980)

114. E. Klempt, B.C. Metsch, C.R. Munz, H.R. Petry, Phys. Lett. B 361, 160 (1995). arXiv:hep-ph/9507449

115. M.G. Olsson, S. Veseli, K. Williams, Phys. Rev. D 52, 5141 (1995). arXiv:hep-ph/9503477

116. J.R. Spence, J.P. Vary, Phys. Rev. C 47, 1282 (1993)

117. A. Martin, Z. Phys. C 32, 359 (1986)

118. M.G. Olsson, S. Veseli, K. Williams, Phys. Rev. D 51, 5079 (1995). arXiv:hep-ph/9410405

119. M.N. Sergeenko, Phys. At. Nucl. 56, 365 (1993) [Yad. Fiz. 56N3, 140 (1993)]

120. M.N. Sergeenko, Z. Phys. C 64, 315 (1994)

121. J.S. Kang, H.J. Schnitzer, Phys. Rev. D 12, 841 (1975)

122. L.K. Sharma, V.P. Iyer, J. Math. Phys. 23, 1185 (1982)

123. C. Goebel, D. LaCourse, M.G. Olsson, Phys. Rev. D 41, 2917 (1990)

124. A. Martin, Phys. Lett. 93B, 338 (1980)

125. E.J. Eichten, C. Quigg, Phys. Rev. D 49, 5845 (1994). arXiv:hep-ph/9402210

126. X.T. Song, H.F. Lin, Z. Phys. C 34, 223 (1987)

127. L. Motyka, K. Zalewski, Z. Phys. C 69, 343 (1996) arXiv:hep-ph/9503420

128. L. Motyka, K. Zalewski, Eur. Phys. J. C 4, 107 (1998). arXiv:hep-ph/9709254 